\title{
STUDDING THE EFFECT OF LIGHTING REGIMENS AND ORGANIC SELENIUM SUPPLEMENTATION ON PRODUCTIVE PERFORMANCE IN DEVELOPMENT LAYING HENS
}

\author{
HASSAN, M. S. H. ${ }^{1}$, HANAN S. M. MOHAMED ${ }^{1}$, I. EL-WARDANY ${ }^{2}$ \\ and A.M.H. AHMED ${ }^{2}$
}

1. Animal Prod. Res. Inst., Agric. Res. Center, Ministry of Agric., Dokki, Giza, Egypt.

2. Poultry production Dept., Fac. of Agric., Ain Shams Univ., Shubra El-Khema, Egypt.

(Manuscript received 8 June 2015)

\begin{abstract}
$\mathrm{T}$ he effect of lighting regimens onproductive performance in two strains of laying hens (Matrouh and Silver Montazah) from 22-42 weeks of age was studied. A total number of 168 hens at 22 weeks of age ( 84 female each) were housed in floor pens. At 24 weeks of age, each strain was randomly distributed to 4 treatments ( 21 hens/ treatment) in 3 replicates 7 hens/ each. The first treatment was served ascontrol group (16hrsL: $8 \mathrm{D})$; the second treatment was subjected to (18 hr. L : 6 D); the third treatment was subjected to (16 hr. L: $8 \mathrm{D})+$ antistress, the last treatment was subjected to (18 hrs L : $6 \mathrm{D})+$ antistress. Where, light treatments (either $16 \mathrm{hr}$. or $18 \mathrm{hr}$. daily) were commenced from 24 to 42 wks of age with constant light intensity of 40 Lux. The antistress was Selenium Yeast (Organic Selenium) at concentration of $0.4 \mathrm{mg} / \mathrm{kg}$ diet.

The obtained results reveal that: Body weight (BW) and growth rate (GR) of Silver Montazah strain were no significantly than Matrouh strain. Also, BW and GR in the treatments that subjected to $18 \mathrm{hr}$. light were lower than that subjected to $16 \mathrm{hr}$ light. The addition of antistress improved BW and GR, but this improvement was not significant. Egg production rate was higher in treatment subjected to $16 \mathrm{hr}$ light compared with $18 \mathrm{hr}$. light, also, it was observed that, also egg production rate was higher in Silver Montazah strain compared with Matrouh strain. The addition of antistress had positive effect on egg production rate. Egg weight was higher in Matrouh strain than Silver Montazah strain, and in treatment subjected to $16 \mathrm{hr}$ compared with $18 \mathrm{hr}$ light. Also the addition of antistress raised the egg weight at all ages. Mortality rate (MR) was higher in the treatment that subjected to $18 \mathrm{hr} \mathrm{light}$ compared with $16 \mathrm{hr}$. light and in Silver Montazah than Matrouh strain, but this increase was not significant. The antistress reduced $M R$, but this reduction was not significant. It is concluded that, using $16 \mathrm{hr}$. light daily and Selenium Yeast supplementation improved the productive performance in development laying hens. Key words: Light period, egg production, developed laying hens.
\end{abstract}

\section{INTRODUCTION}

Light is important for poultry for many reasons. Vision is the predominant sense in birds, where a large proportion of the total brain size is devoted to eyes and 
visual cortex, Güntürkün, (2000). Light as an environmental factor consists of three different aspects: intensity, duration and wavelength. Light intensity, color and the photoperiodic regime can affect the physical activity of broiler chickens.

Moore and Siopes (2000) found that total white blood cell (WBC) counts in Japanese quail increased significantly during a shorter photoperiod (8L: $16 \mathrm{D}$ ) compared to a longer photoperiod (16L:8D) and constant lighting. El-Slamoney, et al. (2010) reported that light treatments ((T1) control, (T2) day light +2 hours artificial light and (T3)day light +4 hours artificial light) had significant effect on body weight gain, egg number, egg production and egg mass at 32-36 weeks of age. Continuous light programs reduce the opportunity for sleep, and sleep deprivation may increase physiological stress. Olanrewaju, et al. (2006) studied the lighting Programs for broiler production, they showed that, light is an important parameter of poultry production. Currently, there are a wide variety of lighting programs (wavelength, intensity, and duration) and devices available to poultry producers, each possessing its own characteristics and applicability to rearing poultry.

Khalil et al. (2008) found that egg mass and egg production were significantly increased for birds exposed to step-up lighting regimen compared to others exposed to other lighting regimens for Manharah and Bandarah hens.

Antioxidants play an important role in maintaining bird health, productive and reproductive performance, Hassan, et al. (2011). Vitamin E, vitamin C and selenium are considered the most important antioxidants to alleviate the deleterious effect of stress Abd-El-Hameid, et al. (2001) and Asli, et al. (2007). Selenium has been recognized as an essential dietary nutrient for over $40 \mathrm{yr}$. It is required for maintenance of health, growth, and physiological functions. Traditionally, Se has been added to poultry diets via inorganic sources, such as sodium selenite ( $\mathrm{Na2SeO3).}$ Recent studies have shown that organic Se is more bioavailable than Se in sodium selenite, Therefore, organic sources of Se, such as Se yeast, have been explored as an alternative to inorganic supplementation, Payne, et al. (2005). Selenium yeast is produced by the fermentation of yeast (Saccharomyces Cerevisiae) in a high Se medium, Maysa (2009).

Chun, et al. (2009) reported that added $0.4 \mathrm{mg}$ of yeast selenium $/ \mathrm{kg}$ diet improved feed efficiency, promoted conversion of thyroxine (T4) to triiodothyronine (T3), minimized the changes of blood biochemical parameters; furthermore, it elevated antioxidases activities and decreased lipid peroxidation products in stressed broilers, it was conclude that high level of organic selenium was able to attenuate stress and oxidative injury. 
Arpasova, et al. (2009) showed that the supplementation of selenized yeast $(0.4 \mathrm{mg} / \mathrm{kg} \mathrm{DM})$ had a significant effect on the concentration of Se in blood of laying hens, also egg weight and egg albumen weight were significantly higher with SY supplementation. Sara, et al. (2008) reported that the supplementation of hen's food with organic selenium not only improves their health and productivity, but can also be a natural way to produce functional food, respectively the production of eggs enriched with selenium.

Salwa, et al. (2004) reported that adding Se had a positively effect on the egg production, egg weight, feed efficiency, weight gain, fertility, chick weight and mortality rate of hens. .direct mode of action of organic selenium which enhanced function of the selenium dependent GSH-px antioxidant system, on the other hand, it was observed that selenium yeast reduced deterioration of the albumen, which results in slower carbon dioxide loss and thus maintains albumen quality after the egg is laid.

The present work was designed to study the effect of lighting regimens and organic selenium supplementation on productive performance in two development laying hens.

\section{MATERIALS AND METHODS}

The present study was carried out at the poultry breeding station "Inshas station", Animal Production Research Institute "APRI", Agricultural Research Center, Ministry of Agriculture, Giza, during the period from June to October. A total number of 168 hens at 22 weeks of age from Silver Montazah and Matrouh strains ( 84 female each) were housed in floor pens (the pen was height 2.5 matter, the long 2 matter,florescent constant light intensity 40 Lux and good ventilation), When the birds reach 24 weeks of age, each strain was randomly distributed to 4 treatments ( 21 hen/ treatment), each treatment was divided into 3 replicates ( 7 hens/ replicate), where the treatments were, where the treatments were:

The first treatment was severed as a control $(16 \mathrm{hr} \mathrm{L}+8 \mathrm{D})$; the second treatment was subjected to $(18 \mathrm{hr} L+6 \mathrm{D})$; the third was subjected to $16 \mathrm{hrs}$ light + antistress and the last treatment was subjected to $18 \mathrm{hrs}$ light + antistress.

Light treatments (either 16 hr. or 18 hr. daily) were continuous from 24 to 42 wks of age with constant light intensity (40 Lux)by using thermostat in all experimental rooms.

From 24 weeks of age and upwards, two lighting regimens were applied until 42 wk of age.

A 16 hrs light as control and 18 hrs light as long photoperiod were provided. Also, a heat exposure treatment were was applied at 24 and 38 wks of age, whirs 
birds were exposed to $39 \pm 1^{\circ} \mathrm{C}$ for 4 hours $L$ day for 4 consecutive days from 10.00 A.M. to 2.00 P.M. using gas heaters. The temperature of the control treatment was the normal ambient temperature ( from 24 to $26^{\circ} \mathrm{C}$ ). Electric fans were used to maintain the indoor temperature within this range and relative humidity was $55-60 \%$. Selenium Yeast (Organic Selenium) was added to developed laying hens rations with a concentration of $0.4 \mathrm{mg} / \mathrm{kg}$ diet from 24 wks of age upwards and used as an antistress compound.

The composition and calculated analysis of the basal diet are shown in Table (1).

\section{Studied traits}

\section{(a) Growth performance}

Body weight, growth Rate (GR), feed intake and mortality rate were recorded monthly.

Where as: $\quad \mathbf{G R}=[($ final body weight-initial body weight $) /\{($ final body weight + initial body weight) / 2\}]*100.

\section{(b) Egg production}

1- Egg weight was recorded daily during the experimental period.

2- Egg production rate were calculated monthly during the experimental period.

Where as: Egg Rate = Egg number / hen/month / (number of days of the same period $x$ number of hens) $x 100$.

Egg quality traits (egg weight $(\mathrm{g})$, egg length $(\mathrm{cm})$, egg width $(\mathrm{cm})$, yolk height(mm), thick albumin height $(\mathrm{mm})$, shell weight(g), shell thickness(Mm), yolk diameter(Mm), yolk color, yolk weight(g) and Haugh unit \% ) was recorded at 38 week of age and using 120 eggs (4 treatments $\times 2$ strains $\times 15$ eggs )

Where as: Haugh units (H.U.) $=100 \log \left(H+7.57-1.7 \mathrm{~W}^{0.37}\right)$.

\section{Statistical analysis}

Collected data were statistically analyzed using the multiple way analyses of variance using the general linear models (GLM) statistical analysis (SAS) software package (2003).

\section{RESULTS AND DISCUSSION}

\section{1- Effect of light and antistress (selenium yeast) on body weight, growth rate and feed intake}

Tables (2 and 3) showed that Silver Montazah strain was insignificantly higher than Matrouh strain in its body weight (BW) and growth rate (GR), also, it showed that BW and GR in birds subjected to $16 \mathrm{hr}$ light was higher hand those subjected to $18 \mathrm{hr}$ light, but this raised was not significant. The addition of antistress had positive 
effect on BW and GR. These results agree with Enaiat, et al., (2009) who reported that Silver Montazah strain had significantly higher body weight, feed consumptionthan Matrouh strain, while there were no significant differences between both strains in age at sexual maturity; El-Slamoney, et al. (2010) who showed that treatments of light ( either day light +2 hours artificial light or day light +4 hours artificial light) had significant effect on body weight and body weight gain. Also,Chun, et al., (2009) studied the effects of different Sources (sodium selenite or yeast selenium) and levels (0, 0.1 or $0.4 \mathrm{mg} \mathrm{Se} / \mathrm{kg}$ of each) on Performance, thyroid function and antioxidant status in stressed broiler chickens, they reported that supplementation of dietary selenium improved feed efficiency, promoted conversion of thyroxine (T4) to triiodothyronine (T3), minimized the changes of blood biochemical parameters; furthermore, it elevated antioxidases activities and decreased lipid peroxidation products in stressed broilers. The effects of adding $0.4 \mathrm{mg} / \mathrm{kg}$ selenium were better than $0.1 \mathrm{mg} / \mathrm{kg}$. Although there was no significant difference between two selenium sources, it was conclude that high level of inorganic or organic selenium was able to attenuate stress and oxidative injury due to exogenous CORT administration, especially organic yeast selenium. Maysa, et al., (2009) reported that, live body weight and growth rate were significantly increased for hens fed Sel-Plex ${ }^{\mathrm{TM}}$ supplementation compared with those in control group.

The effects of strain, lighting regimens and selenium yeast on feed intake are shown in Table (4). Feed intake was higher in Silver Montazah than Matrouh strain at all ages, but the difference was not significant. The 18 hrs light program insignificantly reduced feed intake at all ages compared with $16 \mathrm{hrs}$ light regimen. Moreover, addition of selenium yeast insignificantly elevated feed intake at all ages compared with the control treatment (16 hr light).

\section{2- Effect of light and anti stress (selenium yeast) on egg production rate, egg weight, egg quality and mortality rate.}

Table (4) showed the egg production rate, where we can notice that, egg production rate was higher in Silver Montazah strain compared with Matrouh strain, and treatment which subjected to $16 \mathrm{hr}$ photoperiod was higher than those of $18 \mathrm{hr}$ in the egg rate, also, the anti stress raised the egg rate, but this elevation was not significant. Table (5) showed egg weight of hens where, Matrouh strain was higher in egg weight than Silver Montazah strain. The treatment subjected to $16 \mathrm{hr}$ light was higher than those subjected to $18 \mathrm{hr}$ light. Also the anti stress raised egg weight of hens. Table (6) showed mortality rate, where, Silver Montazah strain was higher than Matrouh strain in mortality rate, also treatment with $18 \mathrm{hr}$ light was higher than $16 \mathrm{hr}$ light in mortality rate. The obtained results agree with Enaiat, et al., (2009) who 
reported that Silver Montazah strain had significantly higher egg number, egg mass, rate of laying and feed consumption than Matrouh strain, while there were no significant differences between both strains in age at sexual maturity; El-Slamoney, et al. (2010) who showed that treatments of light (either day light +2 hours artificial light or day light +4 hours artificial light) had significant effect on egg number, egg production and egg mass at 32-36 weeks of age. Also, Khalil et al. (2008) found that egg mass and egg production were significantly increased for birds exposed to stepup lighting regimen compared to others exposed to other lighting regimens for Manharah and Bandarah hens. Arpasova, et al., (2009) studied the effects of dietary supplementation of sodium selenite and selenized yeast (at dose of $0.4 \mathrm{mg} / \mathrm{kg} \mathrm{DM}$ in the form of SS or SY) on selected qualitative parameters of laying hens eggs, they reported that The supplementation of both forms of selenium into diet had a significant effect on the concentration of Se in blood of laying hens.

Egg weight and egg albumen weight were significantly higher in the experimental groups with SY supplementation only. The supplementation of both forms of selenium into the diet of laying hens significantly affected the width of egg, egg albumen high, Haugh units (HU), egg yolk weight as well as egg yolk ratio. The results indicated that, selenium supplementation into laying hens' basal diet significantly influences the concentration of Se in blood of laying hens and most of the physical qualitative parameters of eggs. Salwa, et al., (2004) investigated that adding Se had a positively effect on the egg production, egg weight, feed efficiency, weight gain, fertility, chick weight and mortality rate of hens. Rutz et al., (2005) and Maysa, et al., (2009) reported that live body weight, egg production percentage, egg weight, egg quality and selenium content in yolk and albumen were significantly increased for hens fed Sel-Plex ${ }^{\mathrm{TM}}$ supplementation compared with those in control group. Sel-Plex ${ }^{\mathrm{TM}}$ supplementation significantly increased the concentrations of total protein, globulin, glutathione peroxidase, selenium, triiodotherionine (T3), hemoglobin( $\mathrm{Hb})$, red blood cells and white blood cells. Also, Renema (2004) and Sara et al., (2008) indicated that the use of organic selenium source improved egg production and egg weight.

It could be concluded that using 16 hours of light per day and the dietary supplementation of antistress (selenium yeast) give almost similar results to improve live body weight, egg production rate, egg weight, give good egg quality and decrease feed intake and mortality rate and improved some productive performance parameters of development laying hens. 
Table 1. Composition and calculated chemical analysis of the basal diet:

\begin{tabular}{|c|c|}
\hline Ingredients & $\%$ \\
\hline Yellow corn & 63.50 \\
\hline Soybean meal (44\%) & 24.57 \\
\hline Wheat bran & 2.0 \\
\hline Limestone (CaCo3) & 7.77 \\
\hline Premix * & 0.30 \\
\hline $\mathrm{NaCl}$ & 0.30 \\
\hline Di-Calcium phosphate & 1.50 \\
\hline DL- methionine & 0.06 \\
\hline Total & 100.00 \\
\hline \multicolumn{2}{|l|}{ Calculated analysis $* *$} \\
\hline $\mathrm{CP}$ & 16.00 \\
\hline M.E. Kcal/kg diet & 2703.34 \\
\hline Crude fiber \% & 3.47 \\
\hline Crude fate \% & 2.86 \\
\hline Calcium \% & 3.32 \\
\hline Available Phosphorus \% & 0.406 \\
\hline Lysine \% & 0.889 \\
\hline Methionine \% & 0.350 \\
\hline Methionine + Cystine & 0.620 \\
\hline Sodium & 0.135 \\
\hline
\end{tabular}

* Premix provides by $3 \mathrm{Kg}$ : Vit. A, $10000000 \mathrm{IU}$; Vit.D3, $2000000 \mathrm{IU}$; Vit. E,10000 IU; Vit K3 1000 mg; Vit.B1 1000 mg; Vit. B2 5000 mg; Vit. B6 1500 mg; Vit. B12, 10 mg, Pantothenic acid 10000 mg; Niacin 30000 mg; Biotin 50 mg; Folic acid 1000 mg; Choline 250 mg; Selenium 100 mg; Copper 4000 mg; Iron 30000 mg; Manganess 60000 mg; Zinc $50000 \mathrm{mg}$; Iodine $1000 \mathrm{mg}$; Cobalt $100 \mathrm{mg}$ and CaCo3 $3000 \mathrm{~g}$.

** According to feed composition tables for animal and poultry feedstuffs used in Egypt (2001).

Table 2. Effect of light regimens and Selenium yeast supplementation on body weight (gm)for two strains of developed laying hens.

\begin{tabular}{|c|c|c|c|c|c|c|}
\hline \multicolumn{2}{|c|}{ Main effect } & 26 wk. age & $30 \mathrm{wk}$. age & 34 wk. age & 38 wk. age & 42 wk. age \\
\hline \multirow{2}{*}{ 1-Strain } & $\begin{array}{l}\text { 1- Silver } \\
\text { Montazah }\end{array}$ & $\begin{array}{c}1187.89 \pm \\
9.0^{\mathrm{a}}\end{array}$ & $\begin{array}{c}1228.40 \pm \\
8.8^{\mathrm{a}}\end{array}$ & $\begin{array}{c}1280.61 \pm \\
9.1^{\mathrm{a}}\end{array}$ & $\begin{array}{c}1340.21 \pm \\
9.59^{\mathrm{a}}\end{array}$ & $\begin{array}{c}1400.50 \pm \\
9.73^{\mathrm{a}} \\
\end{array}$ \\
\hline & $\begin{array}{l}2- \\
\text { Matrouh }\end{array}$ & $\begin{array}{c}1050.98 \pm \\
9.0^{\mathrm{b}}\end{array}$ & $\begin{array}{r}1140.70 \\
8.7^{\mathrm{b}} \\
\end{array}$ & $\begin{array}{c}1200.98 \\
8.9^{b}\end{array}$ & $\begin{array}{c}1255.94 \pm \\
9.59^{\mathrm{b}}\end{array}$ & $\begin{array}{c}1320.37 \pm \\
9.71^{b}\end{array}$ \\
\hline \multirow{2}{*}{ 2- Light } & $1-16 \mathrm{hr}$ & $\begin{array}{r}1140.34 \pm \\
9.0^{\mathrm{a}} \\
\end{array}$ & $\begin{array}{c}1189.13 \pm \\
8.7^{\mathrm{a}} \\
\end{array}$ & $\begin{array}{c}1243.06 \pm \\
8.9^{\mathrm{a}} \\
\end{array}$ & $\begin{array}{c}1294.31 \pm \\
9.40^{\mathrm{a}} \\
\end{array}$ & $\begin{array}{c}1383.89 \pm \\
9.54^{\mathrm{a}} \\
\end{array}$ \\
\hline & $2-18 \mathrm{hr}$ & $\begin{array}{r}1100.39 \pm \\
9.0^{\mathrm{a}} \\
\end{array}$ & $\begin{array}{c}1140.14 \pm \\
8.8^{\mathrm{a}} \\
\end{array}$ & $\begin{array}{c}1207.43 \pm \\
9.1^{a}\end{array}$ & $\begin{array}{c}1240.22 \pm \\
9.74^{\mathrm{a}} \\
\end{array}$ & $\begin{array}{c}1360.54 \pm \\
9.90^{\mathrm{a}}\end{array}$ \\
\hline \multirow{2}{*}{$\begin{array}{l}\text { 3- Anti-stress } \\
\text { (Selenium } \\
\text { Yeast) }\end{array}$} & 1-with & $\begin{array}{c}1140.39 \pm \\
9.0^{\mathrm{a}}\end{array}$ & $\begin{array}{c}1200.42 \pm \\
8.7^{\mathrm{a}}\end{array}$ & $\begin{array}{c}1264.83 \pm \\
8.9^{\mathrm{a}}\end{array}$ & $\begin{array}{c}1301.75 \pm \\
9.54^{\mathrm{a}}\end{array}$ & $\begin{array}{c}1390.43 \pm \\
9.74^{\mathrm{a}}\end{array}$ \\
\hline & 2-without & $\begin{array}{c}1100.48 \pm \\
9.0^{\mathrm{a}}\end{array}$ & $\begin{array}{c}1154.59 \pm \\
8.8^{\mathrm{a}}\end{array}$ & $\begin{array}{c}1210.76 \pm \\
9.1^{\mathrm{a}}\end{array}$ & $\begin{array}{c}1234.40 \pm \\
9.60^{a}\end{array}$ & $\begin{array}{c}1300.44 \pm \\
9.71^{\mathrm{a}}\end{array}$ \\
\hline
\end{tabular}

$a, b \ldots$, means within column with different superscripts are significantly different $(P \leq 0.05)$. 
Table 3. Effect of light regimens and Selenium yeast supplementation on growth rate $\%$ for two strains of developed laying hens.

\begin{tabular}{|l|l|c|c|c|c|}
\hline \multicolumn{2}{|c|}{ Main effect } & $26-30 \mathrm{wk}$ & $30-34 \mathrm{wk}$ & $34-38 \mathrm{wk}$ & $38-42 \mathrm{wk}$ \\
\hline \multirow{2}{*}{ 1-Strain } & $\begin{array}{l}\text { 1- Silver } \\
\text { Montazah }\end{array}$ & $4.94 \pm 0.23^{\mathrm{a}}$ & $5.18 \pm 0.30^{\mathrm{a}}$ & $6.92 \pm 0.07^{\mathrm{a}}$ & $7.09 \pm 0.30^{\mathrm{a}}$ \\
\cline { 2 - 6 } & 2- Matrouh & $4.42 \pm 0.23^{\mathrm{a}}$ & $4.68 \pm 0.30^{\mathrm{a}}$ & $6.48 \pm 0.07^{\mathrm{a}}$ & $6.38 \pm 0.30^{\mathrm{a}}$ \\
\hline \multirow{2}{*}{ 2- Light } & $1-16 \mathrm{hr}$ & $4.90 \pm 0.29^{\mathrm{a}}$ & $5.09 \pm 0.23^{\mathrm{a}}$ & $6.77 \pm 0.31^{\mathrm{a}}$ & $6.88 \pm 0.07^{\mathrm{a}}$ \\
\cline { 2 - 6 } & $2-18 \mathrm{hr}$ & $4.46 \pm 0.30^{\mathrm{a}}$ & $4.76 \pm 0.23^{\mathrm{a}}$ & $6.55 \pm 0.29^{\mathrm{a}}$ & $6.59 \pm 0.07^{\mathrm{a}}$ \\
\hline \multirow{2}{*}{$\begin{array}{l}\text { 3- Anti-stress } \\
\text { (Selenium } \\
\text { Yeast) }\end{array}$} & 1 -with & $4.80 \pm 0.30^{\mathrm{a}}$ & $5.18 \pm 0.23^{\mathrm{a}}$ & $6.70 \pm 0.30^{\mathrm{a}}$ & $6.93 \pm 0.07^{\mathrm{a}}$ \\
\cline { 2 - 6 } & 2 -without & $4.56 \pm 0.30^{\mathrm{a}}$ & $4.68 \pm 0.23^{\mathrm{a}}$ & $6.40 \pm 0.30^{\mathrm{a}}$ & $6.54 \pm 0.07^{\mathrm{a}}$ \\
\hline
\end{tabular}

$a, b \ldots$, means within column with different superscripts are significantly different $(P \leq 0.05)$

Table 4. Effect of light regimens and Selenium yeast supplementation on feed intake ( $\mathrm{g} /$ hen /day) for two strains of developed laying hens.

\begin{tabular}{|l|c|c|c|c|c|}
\hline \multicolumn{2}{|c|}{ Main effect } & $26-30 \mathrm{wk}$ & $30-34 \mathrm{wk}$ & $34-38 \mathrm{wk}$ & $38-42 \mathrm{wk}$ \\
\hline \multirow{2}{*}{1 -Strain } & $\begin{array}{r}\text { 1- Silver } \\
\text { Montazah }\end{array}$ & $114.50 \pm 2.50^{\mathrm{a}}$ & $118.45 \pm 3.30^{\mathrm{a}}$ & $119.50 \pm 3.07^{\mathrm{a}}$ & $121.61 \pm 3.30^{\mathrm{a}}$ \\
\cline { 2 - 6 } & $2-$ Matrouh & $111.07 \pm 2.50^{\mathrm{a}}$ & $112.30 \pm 3.30^{\mathrm{a}}$ & $112.52 \pm 3.07^{\mathrm{a}}$ & $113.92 \pm 3.30^{\mathrm{a}}$ \\
\hline \multirow{2}{*}{ 2- Light } & $1-16 \mathrm{hr}$ & $114.54 \pm 3.51^{\mathrm{a}}$ & $117.05 \pm 3.23^{\mathrm{a}}$ & $116.43 \pm 3.31^{\mathrm{a}}$ & $118.66 \pm 2.97^{\mathrm{a}}$ \\
\cline { 2 - 6 } & $2-18 \mathrm{hr}$ & $111.02 \pm 3.51^{\mathrm{a}}$ & $113.70 \pm 323^{\mathrm{a}}$ & $115.58 \pm 3.31^{\mathrm{a}}$ & $116.87 \pm 2.97^{\mathrm{a}}$ \\
\hline \multirow{2}{*}{ 3- Anti stress } \\
(Selenium
\end{tabular}

$a, b \ldots$, means within column with different superscripts are significantly different $(P \leq 0.05)$

Table 5. Effect of lightregimens and Selenium yeast supplementation on egg production percentage (\%) for two strains of developed laying hens.

\begin{tabular}{|c|c|c|c|c|c|}
\hline \multicolumn{2}{|c|}{ Main effect } & $26-30 \mathrm{wk}$ & $30-34 \mathrm{wk}$ & $34-38 \mathrm{wk}$ & $38-42$ wk \\
\hline \multirow{2}{*}{ 1-Strain } & 1- Silver Montazah & $47.10 \pm 1.93^{\mathrm{a}}$ & $55.12 \pm 0.88^{a}$ & $63.11 \pm 1.22^{a}$ & $68.14 \pm 2.52^{a}$ \\
\hline & 2- Matrouh & $40.90 \pm 1.93^{b}$ & $49.11 \pm 0.88^{b}$ & $56.13 \pm 1.22^{b}$ & $59.10 \pm 2.52^{b}$ \\
\hline \multirow{2}{*}{ 2- Light } & $1-16 \mathrm{hr}$ & $46.54 \pm 1.93^{a}$ & $50.91 \pm 0.88^{a}$ & $53.23 \pm 1.22^{a}$ & $58.20 \pm 2.52^{\mathrm{a}}$ \\
\hline & $2-18 \mathrm{hr}$ & $40.14 \pm 1.93^{b}$ & $43.29 \pm 0.88^{b}$ & $45.55 \pm 1.22^{b}$ & $51.23 \pm 2.52^{b}$ \\
\hline \multirow{2}{*}{$\begin{array}{l}\text { 3- Anti-stress } \\
\text { (Selenium } \\
\text { Yeast) }\end{array}$} & 1-with & $47.20 \pm 1.93^{a}$ & $58.10 \pm 0.88^{a}$ & $60.10 \pm 1.22^{\mathrm{a}}$ & $68.10 \pm 2.52^{\mathrm{a}}$ \\
\hline & 2-without & $40.10 \pm 1.93^{b}$ & $50.13 \pm 0.88^{\mathrm{b}}$ & $57.11 \pm 1.22^{\mathrm{b}}$ & $62.11 \pm 2.52^{b}$ \\
\hline
\end{tabular}

$a, b \ldots$, means within column with different superscripts are significantly different $(P \leq 0.05)$ 
Table 6. Effect of light regimens and Selenium yeast supplementation on egg weight (gm) for two strains of developed laying hens.

\begin{tabular}{|c|c|c|c|c|c|}
\hline \multicolumn{2}{|c|}{ Main effect } & $26-30 w k$ & $30-34$ wk & $34-38 w k$ & $38-42$ wk \\
\hline \multirow{2}{*}{ 1-Strain } & 1- Silver Montazah & $38.29 \pm 0.66^{b}$ & $41.32 \pm 0.15^{b}$ & $46.32 \pm 0.21^{b}$ & $48.12 \pm 2.30^{b}$ \\
\hline & 2- Matrouh & $44.54 \pm 0.66^{a}$ & $46.12 \pm 0.15^{a}$ & $50.31 \pm 0.21^{a}$ & $52.34 \pm 2.30^{a}$ \\
\hline \multirow{2}{*}{ 2- Light } & $1-16 \mathrm{hr}$ & $40.32 \pm 0.66^{a}$ & $43.31 \pm 0.15^{a}$ & $45.34 \pm 0.21^{a}$ & $50.21 \pm 2.30^{a}$ \\
\hline & $2-18 \mathrm{hr}$ & $37.94 \pm 0.66^{a}$ & $40.72 \pm 0.15^{a}$ & $42.22 \pm 0.21^{a}$ & $47.29 \pm 2.30^{a}$ \\
\hline \multirow{2}{*}{$\begin{array}{l}\text { 3- Anti-stress } \\
\text { (Selenium Yeast) }\end{array}$} & 1-with & $38.58 \pm 0.67^{a}$ & $42.99 \pm 0.15^{a}$ & $44.32 \pm 0.21^{a}$ & $47.13 \pm 2.30^{a}$ \\
\hline & 2-without & $36.45 \pm 0.67^{a}$ & $40.21 \pm 0.15^{a}$ & $42.34 \pm 0.21^{a}$ & $45.34 \pm 2.30^{a}$ \\
\hline
\end{tabular}

$a, b \ldots$, means within column with different superscripts are significantly different $(P \leq 0.05)$.

Table 7. Effect of light regimens and Selenium yeast supplementation on egg quality for two strains of developed laying hens.

\begin{tabular}{|l|l|c|c|c|c|}
\hline \multicolumn{2}{|l|}{ Main effect } & \multicolumn{1}{|c|}{$\begin{array}{c}\text { Egg weight } \\
(\mathrm{g})\end{array}$} & $\begin{array}{c}\text { Egg length } \\
(\mathrm{cm})\end{array}$ & $\begin{array}{c}\text { Egg width } \\
(\mathrm{cm})\end{array}$ & $\begin{array}{c}\text { Yolk height } \\
(\mathrm{mm})\end{array}$ \\
\hline \multirow{3}{*}{ 1-Strain } & $\begin{array}{l}\text { 1- Silver } \\
\text { Montazah }\end{array}$ & $41.24 \pm 0.39^{\mathrm{b}}$ & $5.19 \pm 0 . .03^{\mathrm{b}}$ & $3.98 \pm 0.02^{\mathrm{b}}$ & $13.91 \pm 0.04^{\mathrm{a}}$ \\
\cline { 2 - 6 } & $2-$ Matrouh & $45.07 \pm 0.39^{\mathrm{a}}$ & $5.32 \pm 0.03^{\mathrm{a}}$ & $4.09 \pm 0.02^{\mathrm{a}}$ & $14.02 \pm 0.04^{\mathrm{a}}$ \\
\hline \multirow{2}{*}{ 2- Light } & $1-16 \mathrm{hr}$ & $43.46 \pm 0.39^{\mathrm{a}}$ & $5.28 \pm 0.03^{\mathrm{a}}$ & $4.05 \pm 0.02^{\mathrm{a}}$ & $13.99 \pm 0.04^{\mathrm{a}}$ \\
\cline { 2 - 6 } & 2-18 hr & $42.85 \pm 0.39^{\mathrm{a}}$ & $5.22 \pm 0.03^{\mathrm{a}}$ & $4.04 \pm 0.02^{\mathrm{a}}$ & $13.94 \pm 0.04^{\mathrm{a}}$ \\
\hline \multirow{3}{*}{$\begin{array}{l}\text { 3- Anti-stress } \\
\text { (Selenium } \\
\text { Yeast) }\end{array}$} & 1-with & $43.39 \pm 0.39^{\mathrm{a}}$ & $5.26 \pm 0.03^{\mathrm{a}}$ & $4.07 \pm 0.02^{\mathrm{a}}$ & $13.97 \pm 0.04^{\mathrm{a}}$ \\
\cline { 2 - 6 } & 2-without & $42.92 \pm 0.39^{\mathrm{a}}$ & $5.26 \pm 0.03^{\mathrm{a}}$ & $4.00 \pm 0.02^{\mathrm{a}}$ & $13.96 \pm 0.04^{\mathrm{a}}$ \\
\hline
\end{tabular}

$a, b$..., means within column with different superscripts are significantly different $(P \leq 0.05)$.

Table 8. Effect of light regimens and Selenium yeast supplementation on egg quality for two strains of developed laying hens.

\begin{tabular}{|l|l|c|c|c|c|}
\hline \multicolumn{2}{|c|}{ Main effect } & $\begin{array}{c}\text { Thick albumen } \\
\text { height }(\mathrm{mm})\end{array}$ & $\begin{array}{c}\text { Shell weight } \\
(\mathrm{g})\end{array}$ & $\begin{array}{c}\text { Shell thickness } \\
(\mathrm{Mm})\end{array}$ & $\begin{array}{c}\text { Yolk } \\
\text { diameter } \\
(\mathrm{Mm})\end{array}$ \\
\hline \multirow{3}{*}{ 1-Strain } & $\begin{array}{l}\text { 1- Silver } \\
\text { Montazah }\end{array}$ & $5.45 \pm 0.02^{\mathrm{b}}$ & $6.14 \pm 0.05^{\mathrm{b}}$ & $30.57 \pm 0.09^{\mathrm{b}}$ & $4.17 . \pm 0.02^{\mathrm{b}}$ \\
\cline { 2 - 6 } & $2-$ Matrouh & $5.61 \pm 0.02^{\mathrm{a}}$ & $6.46 \pm 0.05^{\mathrm{a}}$ & $30.90 \pm 0.09^{\mathrm{a}}$ & $4.21 \pm 0.02^{\mathrm{a}}$ \\
\hline \multirow{2}{*}{ 2- Light } & $1-16 \mathrm{hr}$ & $5.54 \pm 0.02^{\mathrm{a}}$ & $6.30 \pm 0.05^{\mathrm{a}}$ & $30.79 \pm 0.09^{\mathrm{a}}$ & $4.20 \pm 0.02^{\mathrm{a}}$ \\
\cline { 2 - 6 } & 2-18 hr & $5.51 \pm 0.02^{\mathrm{a}}$ & $6.30 \pm 0.05^{\mathrm{a}}$ & $30.69 \pm 0.09^{\mathrm{a}}$ & $4.19 \pm 0.02^{\mathrm{a}}$ \\
\hline $\begin{array}{l}\text { 3- Anti-stress } \\
\text { Yeast) }\end{array}$ & 1 -with & $5.55 \pm 0.02^{\mathrm{a}}$ & $6.36 \pm 0.05^{\mathrm{a}}$ & $30.74 \pm 0.09^{\mathrm{a}}$ & $4.21 \pm 0.02^{\mathrm{a}}$ \\
\cline { 2 - 6 } & 2-without & $5.50 \pm 0.02^{\mathrm{a}}$ & $6.24 \pm 0.05^{\mathrm{a}}$ & $30.74 \pm 0.09^{\mathrm{a}}$ & $4.17 \pm 0.02^{\mathrm{a}}$ \\
\hline
\end{tabular}

$a, b \ldots$, means within column with different superscripts are significantly different $(P \leq 0.05)$. 
Table 9. Effect of light regimens and Selenium yeast supplementationon egg qualityfor two strains of developed laying hens.

\begin{tabular}{|l|l|c|c|c|}
\hline \multicolumn{2}{|c|}{ Main effect } & Yolk color & $\begin{array}{c}\text { Yolk weight } \\
(\mathrm{g})\end{array}$ & $\begin{array}{c}\text { Haugh Unit } \\
(\%)\end{array}$ \\
\hline \multirow{2}{*}{ 1-Strain } & $1-$ Silver Montazah & $7.48 \pm 0.06^{\mathrm{b}}$ & $16.24 \pm 0.13^{\mathrm{b}}$ & $79.12 \pm 0.10^{\mathrm{b}}$ \\
\cline { 2 - 5 } & 2- Matrouh & $7.56 \pm 0.06^{\mathrm{a}}$ & $17.25 \pm 0.13^{\mathrm{a}}$ & $79.31 \pm 0.10^{\mathrm{a}}$ \\
\hline \multirow{2}{*}{ 2- Light } & $1-16 \mathrm{hr}$ & $7.56 \pm 0.06^{\mathrm{a}}$ & $16.76 \pm 0.13^{\mathrm{a}}$ & $79.29 \pm 0.10^{\mathrm{a}}$ \\
\cline { 2 - 5 } & 2-18 hr & $7.48 \pm 0.06^{\mathrm{a}}$ & $16.73 \pm 0.13^{\mathrm{a}}$ & $79.23 \pm 0.10^{\mathrm{a}}$ \\
\hline \multirow{2}{*}{ 3- Anti-stress } & 1-with & $7.61 \pm 0.06^{\mathrm{a}}$ & $16.89 \pm 0.13^{\mathrm{a}}$ & $79.29 \pm 0.10^{\mathrm{a}}$ \\
\cline { 2 - 5 } (Selenium Yeast) & 2-without & $7.43 \pm 0.06^{\mathrm{a}}$ & $16.59 \pm 0.13^{\mathrm{a}}$ & $79.23 \pm 0.10^{\mathrm{a}}$ \\
\hline
\end{tabular}

$a, b \ldots$, means within column with different superscripts are significantly different $(P \leq 0.05)$.

Table 10. Effect of light regimens and Selenium yeast supplementation on mortality rate (\%) for two strains of developed laying hens.

\begin{tabular}{|c|c|c|c|c|c|}
\hline \multicolumn{2}{|c|}{ Main effect } & $24-28 w k$ & $28-32 w k$ & $32-36 w k$ & $36-40 \mathrm{wk}$ \\
\hline \multirow{2}{*}{ 1-Strain } & 1- Silver Montazah & $1.04 \pm 0.49^{a}$ & $1.00 \pm 0.15^{a}$ & $0.40 \pm 0.77^{a}$ & $0.30 \pm 0.33^{a}$ \\
\hline & 2- Matrouh & $0.42 \pm 0.49^{a}$ & $0.30 \pm 0.15^{a}$ & $0.00 \pm 0.77^{a}$ & $0.00 \pm 0.33^{a}$ \\
\hline \multirow{2}{*}{ 2- Light } & $1-16 \mathrm{hr}$ & $0.42 \pm 0.49^{a}$ & $0.21 \pm 0.15^{a}$ & $0.00 \pm 0.77^{a}$ & $0.00 \pm 0.33^{a}$ \\
\hline & $2-18 \mathrm{hr}$ & $0.83 \pm 0.49^{a}$ & $0.86 \pm 0.15^{a}$ & $0.63 \pm 0.77^{a}$ & $0.42 \pm 0.33^{a}$ \\
\hline \multirow{2}{*}{$\begin{array}{l}\text { 3- Anti-stress } \\
\text { (Selenium Yeast) }\end{array}$} & 1-with & $0.30 \pm 0.49^{a}$ & $0.21 \pm 0.15^{a}$ & $0.00 \pm 0.77^{a}$ & $0.00 \pm 0.33^{a}$ \\
\hline & 2-without & $1.20 \pm 0.49^{a}$ & $1.00 \pm 0.15^{a}$ & $0.63 \pm 0.77^{a}$ & $0.55 \pm 0.33^{a}$ \\
\hline
\end{tabular}

$a, b \ldots$, means within column with different superscripts are significantly different $(P \leq 0.05)$.

\section{REFERENCES}

1. Abd-El-Hameid, E. F.; H.M.Houssein and F.E. Afifi. 2001. Study on herbs, vitamin $\mathrm{C}$ and vitamin $\mathrm{E}$ as feed supplement into broiler diets under summer condition. Egyptian.J. Appl. Sc.; 16 (4).

2. Asli, M.M.; Hosseini S.A.; H. Lotfollahian and F.Shariatmadari. 2007. Effects of probiotics, yeast, vitamin $\mathrm{E}$ and vitamin $\mathrm{C}$ supplements on performance and immune response of laying hens during high environmental temperature. Int. J. Poult. Sci, 6: 895-900.

3. Arpasova,H.; V. Petrovic ; M. Mellen; M. Kacaniova; K. Cobanova and L. Leng. 2009. The effects of supplementing sodium selenite and selenized yeast to the diet for laying hens on the quality and mineral content of eggs. Journal of Animal and Feed Sciences, 18, 90-100. 
4. Chun Fan, Bing Yu and Daiwen Chen. 2009. Effects of different sources and levels of Selenium on Performance, thyroid function and antioxidant status in stressed broiler chickens. International Journal of Poultry Science. Volume 8 Number 6: 583-587.

5. El-Slamoney, A.E.; A.M. Battaa El-Neney; S.F. Hassan; R.E. Abd el-Karim and E.H. Abdulla. 2010. Effect of photoperiod and tryptophan amino acid supplementation on pineal gland hormone (melatonin) and its relation to performance in local strain. 1- Effect on laying hen performance. Egypt. Poult. Sci. vol (30): 927-960.

6. Enaiat, M.M.; A.S. Amina; M. Abou-Eitta, Eman and A.H.A. Al-Kotait. 2009. A comparative study between two local strains under cage and floor housing systems.Egypt. Poult. Sci., Vol (29) (I): 439-464.

7. Feed composition tables for animal and poultry feedstuffs used in Egypt. 2001. TechnicalbulletinNo.1, Central Lab for Feed and Food; Ministry of Agriculture, Egypt.

8. Güntürkün O. 2000. Sensory Physiology: Vision. Chapter 1 in Whittow GC (ed): Sturkie's Avian Physiology, Fifth Edition. Academic Press, San Diego. pp 1-19.

9. Hassan, M.S.H.; O.A. El-Sayed and M.M.M.Namera. 2011. Effect of dietary sodium bicarbonate and potassium chloride supplementation on acid-base balance, plasma electrolytes and Aldosterone hormone of Golden Montazah hens under hot climate condition.Egypt. Poult. Sci. Vol (31) (II): 285-303.

10. Khalil , M., Hanaa; M.; R.E. Rizk ; E.H.A. Shahein ; A.M. Mona and Amany ElSahn. 2008. Studies on the optimum light requirements on productive and reproductive performance of local chicken strains .2- Effect of different lighting regimens on productive and reproductive performance and subsequent posthatch progeny growth of local chicken strains. Egyp.Poult. Sci. Vol (28) (III): 901-923.

11. Maysa, M.H.; A.M.H. El-Sheikh, and E.A. Abdalla. 2009. The effect of organic selenium supplementation on productive and physiological performance in a local strain of chickens. 1-The effect of organic selenium (SEL-PLEX ${ }^{\mathrm{TM}}$ on productive, reproductive and physiological traits of Bandarahlocal strain.Egypt. Poult. Sci. Vol (29) (IV): 1061-1084.

12. Moore, C.B. and T.D. Siopes. 2000. Effects of light conditions and melatonin supplementation on the cellular and humoral immune responses inJapanese quail (Coturnixcoturnix japonica). Gen. Comp. Endocrinol., 119: 95-104. 
13. Olanrewaju , H.A. ; J.P. Thaxton; W.A. Dozie; J. Purswell; W.B. Roush and S.L. Branton. 2006. A Review of lighting programs for broiler production. International Journal of Poultry Science 5 (4): 301-308.

14. Payne, R. L., T. K. Lavergne, and L. L. Southern. 2005. Effect of inorganic versus organic selenium on hens production and selenium concentration. Poult. Sci. 84:232-237.

15. Renema, R.A. 2004. Reproductive responses to Se-Plex organic selenium in male and female broiler breeders : impact on production traits and hatchability. In : Bnutritional Biotechnology in the feed and food industries. Proceedings of 20 thAllech s Annal Symposium. Edited by Lyons. T.P. and K.A. Nottingham University press. Nottingham. UK. PP. 81-91.

16. Rutz, F.; M.A. Anciuti; J.L. Rech and E.G. Xavier. 2005. Following response to Sel- Plex and other organic minerals through the broiler breeder mase: case studies in Brazil. In: Nutritional Biotechnology in the feed and food industries: Proceedings of Allech s 21 st. Annual Symposium (T.P. Lyons and K.A. Jacques .eds). Nottingham University Press. U.K. PP. 55-66.

17. Salwa, S.S.; K.M. Mansour; E.M.M. El-Anwar and A.A. EL-Warith. 2004. Laying hen's performance, hatchability, immune response and some blood constituents as affected by vitamin $\mathrm{E}$ and selenium supplementation under hot condition.Egypt. Poultry Sci., Vol.24(II): 483-496.

18. Sara, A.; M. Bennea; A. Odagiu and L. Panta. 2008. Effects of the organic selenium (Se-Plex) administrated in laying hens feed in second laying phase on productive performances and the eggs quality. Bulletin UASVM Animal Science and Biotechnologies, 65: 1-2.

19. SAS. 2003. Statistical Analysis System, User' S Guide, Statistics.Ver.8.2, SAS Institute Institute., Carry, North Carolina. 


\section{دراسة تأثيرنظم الاضاءه واضافة السلينيوم العضوى على الاداء الانتاجى فى الاجاج البياض المستنبط}

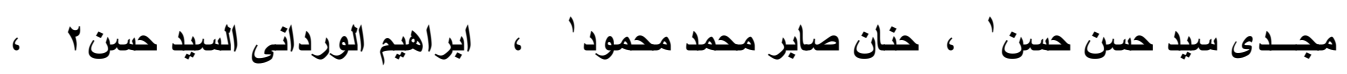
أيمن محمد حسن أحمدץ مدمد مدير

$$
\text { r ـ ـ الجم بحوث تربية الدواجن - معهد بحوث الانتاج الحبوانى - مركز البحوث الزراعبه - الدقى }
$$

أجريت هذه الدراسه بمحطة بحوث تربية الدواجن بأنشاص معهد بحوث الانتاج الحيوانى. و

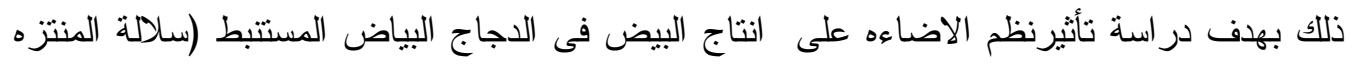

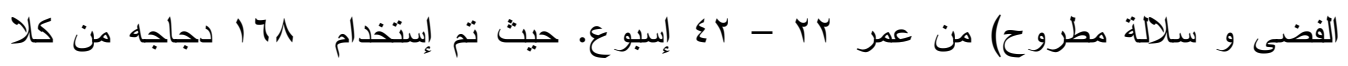

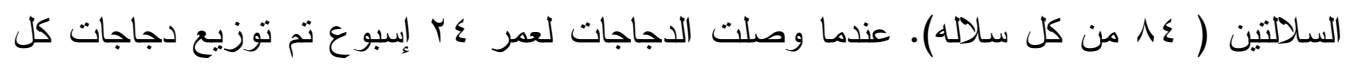

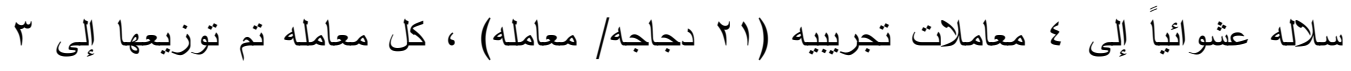

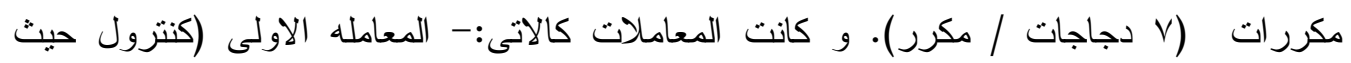

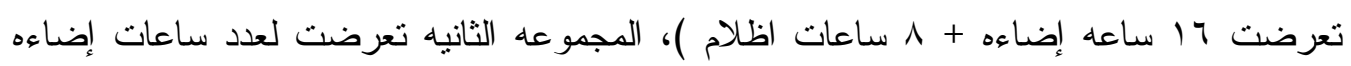

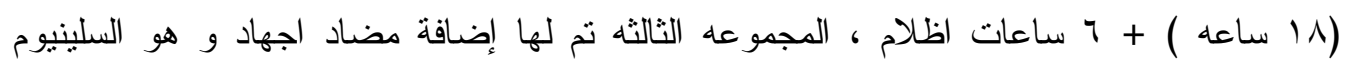

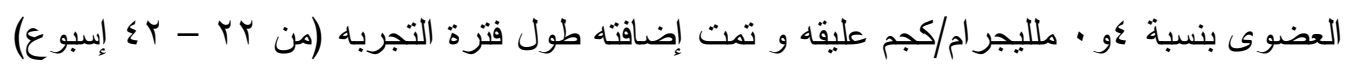

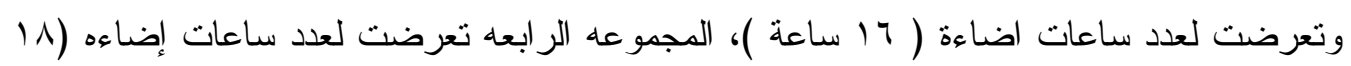

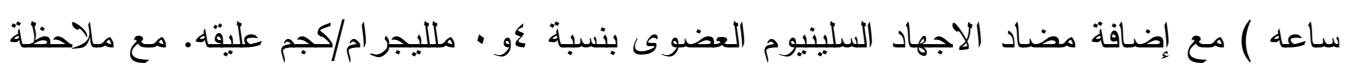

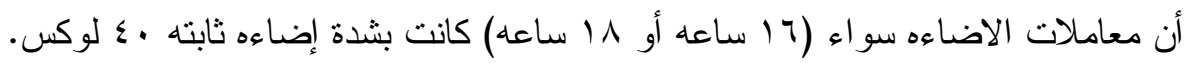

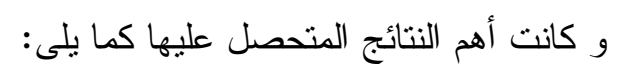

1 - وزن الجسم و معدل النمو و الغذاء المأكول كان أعلى معنويا فى سلالة المنتزه الفضى

$$
\text { بالمقارنه بسلالة مطروح. }
$$

r- معاملة الاضاءه (1) ساعه إضاءه) سببت خفض وزن الجسم و معدل النمو و استهلاك

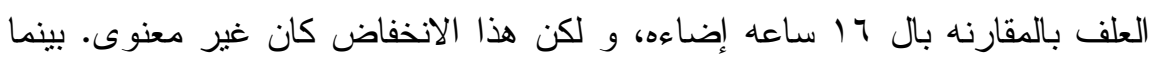

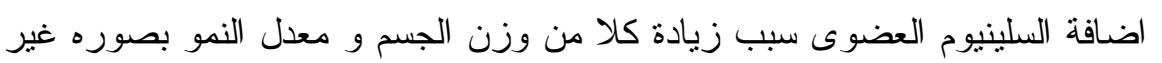

$$
\text { معنوياه. }
$$

r- معدل إنتاج البيض كان أعلى معنويا فى سلالة المنتزه الفضى بالمقارنه بسلالة مطروح

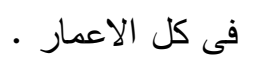

ع - معاملة 1/ ساعه إضاءه سببت إنخفاض معنوى فى معدل انتاج البيض بالمقارنه

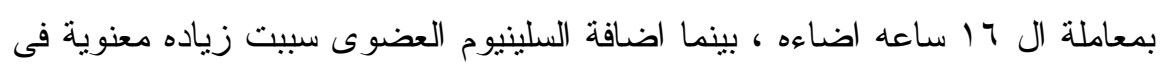
معدلات انتاج البيض فى كل الاعمار . 
هـ ـ وزن البيض فى سلالة مطروح كان أعلى معنويا من المنتزه الفضى فى كل الاعمار .

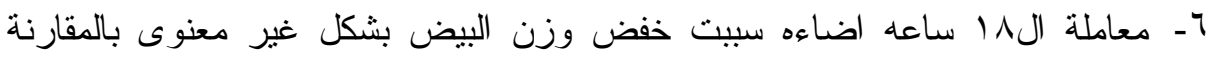

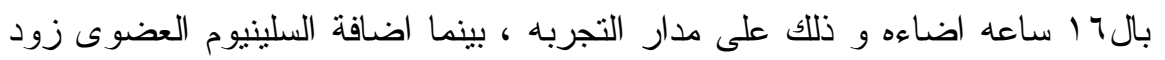

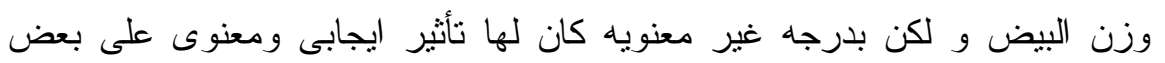

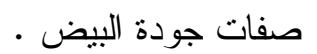
V- معدلات النفوق كانت أعلى فى سلالة المنتزه الفضى بالمقارنه بسلالة مطروح و ذللك بذرجه غير معنويه. ^- معاملة ال 11 ساعه اضاءه كانت بها معدلات النفوق اعلى من معاملة ال 17 ساعه

$$
\text { اضاءهو ولكن بدرجه غير معنوية . }
$$

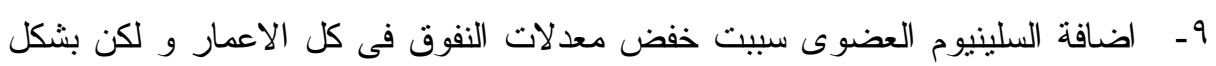

$$
\text { غير معنوى. }
$$

توصى الدراسة باستخدام 17 ساعة اضاءة لكل يوم مع إضافة مضاد الاجهاد السلينيوم العضوى بنسبة ؛و · ملليجرام/كجم عليقه لتحسين الآداء الاتتاجى للاجاج البياض المستنبط. 